\title{
Familial hyperaldosteronism
}

D.J. Torpy ${ }^{1}$,

C.A. Stratakis ${ }^{2}$ and G.P. Chrousos ${ }^{3}$

\author{
'Department of M edicine, Greenslopes Hospital, U niversity of Q ueensland, \\ Brisbane, Australia \\ ${ }^{2}$ Developmental Endocrinology Branch, and ${ }^{3}$ Pediatric and Reproductive Branch, \\ National Institute of Child Health and Development, NIH, Bethesda, MD, USA
}

\section{Correspondence \\ D.J. Torpy \\ Department of Medicine \\ Greenslopes Hospital \\ University of Q ueensland \\ Newdegate Street \\ Brisbane, Q Id, 4120 \\ Australia \\ Presented at the First \\ International Meeting on Adrenal \\ Disease: Basic and Clinical \\ Aspects, Ribeirão Preto, SP, Brazil, \\ August 31-September 2, 1999.}

Received December 20, 1999

Accepted April 13, 2000

\section{Abstract}

Aldosterone, the major circulating mineralocorticoid, participates in blood volume and serum potassium homeostasis. Primary aldosteronism is a disorder characterised by hypertension and hypokalaemia due to autonomous aldosterone secretion from the adrenocortical zona glomerulosa. Improved screening techniques, particularly application of the plasma aldosterone:plasma renin activity ratio, have led to a suggestion that primary aldosteronism may be more common than

Key words

- Aldosterone

- Hyperaldosteronism

- Genetics

- Adrenal tumour

- Hypertension

$\ldots \ldots \ldots \ldots \ldots \ldots . . \ldots \ldots$ previously appreciated among adults with hypertension. Glucocorticoid-remediable aldosteronism (GRA) was the first described familial form of hyperaldosteronism. The disorder is characterised by aldosterone secretory function regulated chronically by ACTH. Hence, aldosterone hypersecretion can be suppressed, on a sustained basis, by exogenous glucocorticoids such as dexamethasone in physiologic range doses. This autosomal dominant disorder has been shown to be caused by a hybrid gene mutation formed by a crossover of genetic material between the ACTH-responsive regulatory portion of the $11 \mathrm{~B}$ hydroxylase (CYP11B1) gene and the coding region of the aldosterone synthase (CYP11B2) gene. Familial hyperaldosteronism type II (FH-II), so named to distinguish the disorder from GRA or familial hyperaldosteronism type I (FH-I), is characterised by autosomal dominant inheritance of autonomous aldosterone hypersecretion which is not suppressible by dexamethasone. Linkage analysis in a single large kindred, and direct mutation screening, has shown that this disorder is unrelated to mutations in the genes for aldosterone synthase or the angiotensin II receptor. The precise genetic cause of FH-II remains to be elucidated.

\section{Introduction}

Aldosterone, the major circulating mineralocorticoid, is a steroid hormone produced exclusively in the zona glomerulosa in a series of six biosynthetic steps. The product of the CYP11B2 gene is capable of catalysing the 11ß-hydroxylase, 18-hydroxylase and 18hydroxydehydrogenase steps in aldosterone biosynthesis $(1,2)$. The CYP11B2 gene is located on human chromosome 8q24.3-tel, in close proximity to the highly homologous CYP11B1 gene, which codes only for the 11ß-hydroxylase enzyme, and catalyses the final step in cortisol synthesis (3). At the level of the zona glomerulosa, the major stimulatory influences are angiotensin II and serum potassium concentration $(4,5)$. 
Adrenocorticotropic hormone (ACTH) stimulates aldosterone secretion in an acute and transient fashion, but ACTH probably does not play a direct role in the chronic regulation of aldosterone secretion $(6,7)$. The major inhibitory influences affecting the zona glomerulosa are exerted by circulating atrial natriuretic peptide (8), and locally, by dopamine (9). Aldosterone acts on its target tissues (distal renal tubule, sweat glands, salivary glands, large intestinal epithelium) via its own specific mineralocorticoid receptors $(10,11)$. Mineralocorticoid receptors exhibit equal affinity for aldosterone and glucocorticoids (12), but distal renal tubular receptors are protected from the effects of cortisol by 113 -hydroxysteroid dehydrogenase, which converts cortisol to inactive cortisone. A number of aldosterone precursors, including deoxycorticosterone and 18-hydroxycorticosterone, have mineralocorticoid activity, and their hypersecretion in various pathological states may produce or exacerbate features typical of mineralocorticoid hypertension.

Hyperaldosteronism is characterised by excessive secretion of aldosterone with consequent increased sodium reabsorption and potassium and hydrogen ion excretion. Clinical features include hypertension, hypokalaemia and metabolic alkalosis. Hyperaldosteronism may alter glucose metabolism through altering insulin secretion, an effect of reduced pancreatic $\beta$-cell intracellular potassium, or altering insulin sensitivity $(13,14)$. Correspondingly, leptin secretion, which is responsive to insulin levels, is reduced in primary aldosteronism and recovers following curative adrenalectomy (15). Hence, it is possible that undiagnosed primary aldosteronism may contribute to the load of type II diabetes mellitus among hypertensives (16). Improved screening methods for primary aldosteronism may allow evaluation of this possibility. In addition, cardiovascular fibrosis has been related to aldosterone hypersecretion (17-19).
Hyperaldosteronism represents a subset of disorders known as mineralocorticoid hypertension. These include two causes of congenital adrenal hyperplasia (11ß-hydroxylase deficiency and $17 \alpha$-hydroxylase deficiency), the syndrome of apparent mineralocorticoid excess due to $11 ß$-hydroxysteroid dehydrogenase deficiency, primary glucocorticoid resistance, Liddle's syndrome, and exogenous sources of mineralocorticoid such as licorice or drugs such as carbenoxolone. The features of mineralocorticoid excess are also often seen in Cushing's syndrome, particularly in patients with ectopic ACTH-producing tumours. In these cases, it is postulated that excessive glucocorticoid levels overwhelm the ability of the 11ß-hydroxysteroid dehydrogenase enzyme to inactivate cortisol at the kidney mineralocorticoid receptor level.

\section{Primary aldosteronism}

Primary aldosteronism was originally described by J.W. Conn (20) as a syndrome of hypertension associated with hypokalaemia and postulated hypersecretion of an endogenous mineralocorticoid. Early reported cases were due to an aldosterone-producing adrenocortical adenoma (APA), removal of which led to some of the earliest cures of hypertension (21).

Earlier reliance on plasma potassium as a screening test, as advocated by some authorities (22), may have led to under-recognition of the contribution of primary aldosteronism to hypertension. An early study using saline infusion as a screening test for primary aldosteronism reported a frequency of $2.2 \%$ of primary aldosteronism among 1,036 unselected hypertensives (23), but recent studies using the ratio of plasma aldosterone to plasma renin activity (ARR) concentrations in plasma suggested that primary aldosteronism may account for at least $8.5 \%$ of hypertensives $(24,25)$. The use of laparoscopic adrenalectomy has reduced the mor- 
bidity of surgery for APA (26). Primary aldosteronism is most frequently diagnosed in middle-aged adults, is more common in women, and rare in children.

\section{Diagnosis of primary aldosteronism}

The currently advocated diagnostic process follows the sequence of screening, confirmation of aldosterone secretory autonomy, differential diagnosis (unilateral surgically remediable or bilateral source of aldosterone), and exclusion of familial hyperaldosteronism type I (27). The validity of screening for primary aldosteronism with the ARR, performed under random conditions with respect to salt intake is now well documented (28-30). However, there is no consensus regarding the indications for screening among hypertensives which takes into account recent reports of increased prevalence of primary aldosteronism using the ARR. The presence of hypertension and hypokalaemia predicts primary aldosteronism with $50 \%$ accuracy (31), however normokalaemic hypertensive primary aldosteronism is now well recognised. Factors which may lead to misleading ARR tests need to be taken into account, including diuretics, spironolactone, ß-blockers, dihydropyridine calcium channel blockers, renal impairment and untreated hypokalaemia. Several tests have been advocated for confirmation of aldosterone secretory autonomy including the intravenous saline suppression test (23), the oral salt/ fludrocortisone suppression test (25), the oral salt loading test with measures of 24-h urine aldosterone excretion (32), or the oral captopril test $(33,34)$. These tests have not been rigorously compared. In some cases with very high ARR tests, confirmation of aldosterone secretory capacity may not be necessary.

Differential diagnosis, primarily between bilateral adrenal hyperplasia (BAH) and APA, can be achieved with postural testing, where $\mathrm{BAH}$ exhibits a rise in aldosterone with upright posture indicative of responsiveness to angiotensin II (35), 18-hydroxycorticosterone levels $(35,36)$, adrenal computed tomograms ( $70 \%$ sensitivity for APA, coexistent incidentalomas may confound this test) and adrenal venous sampling, which remains the gold standard test despite some controversy over technique and interpretation $(37,38)$. Uncommon forms of primary aldosteronism include angiotensin II responsive APA, which show an aldosterone response to posture or angiotensin II infusion, glucocorticoid-remediable aldosteronism which can be excluded with specific genetic testing or a dexamethasone suppression test, and primary adrenal hyperplasia, where unilateral autonomous aldosterone secretion occurs without a discrete tumour on gland resection $(38,39)$.

Familial forms of hyperaldosteronism include familial hyperaldosteronism type I (FH-I) (glucocorticoid-remediable aldosteronism) and familial hyperaldosteronism type II (FH-II). Hyperaldosteronism has also been seen in multiple endocrine neoplasia type 1 (MEN-I) (40).

\section{Familial hyperaldosteronism type I}

In 1966, the first familial cases of hypertension due to a dexamethasone suppressible form of hyperaldosteronism were reported (41). This autosomal dominant disease is known as glucocorticoid-remediable aldosteronism (GRA) or recently, FH-I. GRA is characterised by bilateral adrenal hyperplasia, or rarely, adrenal adenoma (42). GRA accounts for approximately $1 \%$ of cases of primary hyperaldosteronism.

The genetic locus for this disorder was recently established by genetic linkage analysis and the causative mutation subsequently identified (43). The defect involves a crossover of genetic material between the closely related highly homologous genes that code for the enzyme 11ß-hydroxylase (CYP11B1) (which catalyses the last step in cortisol bio- 
synthesis) and the gene for aldosterone synthesis (CYP11B2). Fusion of a portion of the regulatory region of CYP11B1, the ACTHresponsive promoter, with the coding region of the CYP11B2 gene allows aldosterone synthesis to be strongly directed by ACTH, resulting in pathologically high levels of aldosterone and extreme suppressibility of aldosterone to exogenous glucocorticoid administration.

Elucidation of this mutation readily explained the cardinal pathophysiological features of the disorder. The synthesis of "hybrid" steroids, 18-oxocortisol and 18-hydroxycorticosterone, requires the action of $17 \alpha$-hydroxylase on aldosterone, an enzyme expressed only in the zona fasciculata, suggesting that aldosterone is synthesised in the zona fasciculata in this disorder (44). Higher blood pressure in the offspring of affected mothers rather than affected fathers has been attributed to the effects of high maternal aldosterone on the fetus rather than imprinting $(45,46)$. Importantly, not all individuals with the GRA mutation have hypertension, and ongoing studies of the physiologic and genetic bases of their counterbalancing hypotensive systems are in progress (47). The lack of hypokalaemia in many subjects with GRA has been related to a blunted aldosterone response to potassium, which may reduce the severity of hyperaldosteronism (48). Availability of a genetic test for GRA has allowed exploration of the phenotype, such as demonstration of the association of intracranial aneurysm and haemorrhagic stroke with GRA (49).

\section{Familial hyperaldosteronism type II}

Familial hyperaldosteronism type II as a distinct entity was recognised by R.D. Gordon $(25,50)$, although other isolated familial cases of non-dexamethasone suppressible hyperaldosteronism have also been reported $(51,52)$. In addition, the author is aware of several other families with non-dexametha- sone suppressible familial hyperaldosteronism, from a variety of other countries. After the initial serendipitous discovery of two cases in a single family, subsequent cases have been identified by application of the ARR screening test followed by confirmatory testing, and surgery if indicated. To date, 17 families (44 individuals) with FH-II have been identified by the Gordon group (50). All families are of Caucasian descent, from a variety of European backgrounds. There has been no clinical or biochemical evidence of multiple endocrine neoplasia type I in the affected families. Families with APA alone, BAH or both entities represented in one family have been detected. The age range at diagnosis was 14-72 years.

The FH-II phenotype is clinically indistinguishable from a large control group of cases of sporadic primary aldosteronism with respect to age of onset, gender, frequency of hypokalaemia, aldosterone and plasma renin activity levels, and relative frequency of APA and idiopathic hyperaldosteronism (50). In contrast to FH-I, for which genetic testing is available and autosomal dominant inheritance is well established, the mode of inheritance of FH-II remains speculative. However, in the large kindred reported elsewhere, vertical transmission suggests autosomal dominant inheritance. This is also the case in six other FH-II families. In the largest FH-II kindred reported, X-linked inheritance cannot be excluded on segregation analysis, although linkage analysis involving microsatellites on the X-chromosome have been negative.

Our genetic studies of FH-II have focussed on a single large kindred with seven family members who suffer from hyperaldosteronism. Hyperaldosteronism has been demonstrated by the use of the ARR in all cases, and fludrocortisone suppression testing in six of the seven affected individuals. The five hypertensive subjects with primary aldosteronism had adrenal venous sampling results indicative of bilateral adrenal autono- 
mous aldosterone production. Hence, adrenalectomy was not justified and tumour tissue was not available from this family. For genetic linkage analysis purposes, those with positive ARR tests and nonsuppression of aldosterone levels on fludrocortisone suppression testing were classified as affected, hypertensive individuals with normal ARR or those younger than 20 were classified a "query" affected, and normotensive individuals with normal ARR and age greater than 20 were classified as unaffected (50).

A number of candidate genes were studied in terms of physiologically based hypotheses regarding the possible aetiology of FH-II. These hypotheses included the possibility of activating mutations of the angiotensin II type 1 (AT1) receptor gene and the CYP11B2 "aldosterone synthase" gene. We excluded involvement of the AT1 receptor gene in FH-II by means of genetic linkage analysis using a dinucleotide (CA) repeat polymorphism contained in a $0.9-\mathrm{kb}$ EcoRI fragment approximately $15 \mathrm{~kb}$ downstream from the $5^{\prime}$ end of the AT1 gene coding sequence (53).

Although the hybrid gene mutation which causes FH-I had tested negative by PCR (54), and/or Southern blot technique in all cases, an activating mutation of CYP11B2, different from the hybrid gene mutation seen in FH-I, could cause FH-II (55). Accurate chromosomal localisation of the CYP11B2 gene was achieved by radiation hybrid mapping and fluorescent in situ hybridisation (3). We then excluded involvement of the CYP11B2 gene in the FH-II phenotype of the single large kindred using a poly-A repeat marker within the 5 ' region of the CYP11B2 gene and nine other markers in an 80 -cm region extending from $8 \mathrm{q} 21-8 \mathrm{qtel}(50)$. The LOD (logarithm of the odds) score for the CYP11B2 intragenic marker was -12.6 at a recombination distance (2) of 0 .

A number of other candidate genes were excluded from segregation with the FH-II phenotype in this family, including the MENI locus at chromosome 11q13. Although loss of heterozygosity at the $11 \mathrm{q} 13$ chromosomal locus has been described in APA, including those from FH-II patients, loss of heterozygosity at this locus is a frequent finding in sporadic endocrine tumours. Currently, a genome-wide search is near completion where the genetic locus for FH-II should be identified. Elucidation of the causative gene in FH-II may have implications for both sporadic primary aldosteronism and hypertension in general.

\section{Conclusions}

Familial primary aldosteronism has offered opportunities to understand the genetic mechanisms underlying at least a subset of primary aldosteronism. This may be of importance in sporadic primary aldosteronism, which is being recognised with increasing frequency due to the application of plasma ARR testing in normokalaemic hypertensives. The advent of laparoscopic adrenalectomy has reduced the morbidity of surgical treatment of primary aldosteronism. The unremarkable clinical features of FH-II so far ascertained relative to sporadic primary aldosteronism, suggest that biochemical screening of family members of primary aldosteronism patients should be employed more widely. With increasing recognition, a need to optimise diagnostic strategies will become evident. The place of screening for primary aldosteronism among hypertensives has not yet received critical cost-benefit analysis. 


\section{References}

1. Mornet E, Dupont J, Vitek A \& White PC (1989). Characterisation of the two genes encoding human steroid 11ß-hydroxylase (P-450 (11)ß). J ournal of Biological Chemistry, 264: 20961-20967.

2. Curnow KM, Tusie-Luna MT, Pascoe L, Natarajan R, Gu J L, Nadler J L \& White PC (1991). The product of the CYP11B2 gene is required for aldosterone biosynthesis in the human adrenal cortex. Molecular Endocrinology, 5: 1513-1522.

3. Taymans SE, Pack S, Pak E, Torpy DJ, Zhuang Z \& Stratakis CA (1998). Human CYP11B2 (aldosterone synthase) maps to chromosome 8q24.3. J ournal of Clinical Endocrinology and Metabolism, 83: 10331036.

4. McKenna TJ, Island DP, Nicholson WE \& Liddle GW (1978). The effect of potassium on early and late steps in aldosterone biosynthesis in cells of the zona glomerulosa. Endocrinology, 103: 14111416.

5. Clyne $C D$, Zhang $Y$, Slutsker $L$, Mathis J M, White PC \& Rainey WE (1997). Angiotensin II and potassium regulate human CYP11B2 transcription through common cis-elements. Molecular Endocrinology, 11: 638-649.

6. Brown J J , Davies DL \& Lever AF (1964). Variations in plasma renin concentration in several physiological and pathological states. J ournal of the Canadian Medical Association, 90: 201-206.

7. Gaillard RC, Riondel AM, Farrod-Coune CA, Valloton MB \& Muller AF (1983). Aldosterone escape to chronic ACTH administration in man. Acta Endocrinologica, 103: 116-124.

8. Blair-West J R, Coughlin J P, Denton DA, Fei DT, Hardy KJ , Scoggins BA \& Wright RD (1980). A dose response comparison of the actions of angiotensin II and III in sheep. J ournal of Endocrinology, 87: 409417.

9. Carey RM (1982). Acute dopaminergic inhibition of aldosterone secretion is independent of angiotensin II and adrenocorticotropin. J ournal of Clinical Endocrinology and Metabolism, 54: 463-469.

10. Sheppard KE \& Funder J W (1987). Equivalent affinity of aldosterone and corticosterone for type I receptors in kidney and hippocampus: direct binding studies. J ournal of Steroid Biochemistry, 28: 737742.

11. Sheppard KE \& Funder J W (1987). Type I receptors in parotid, colon, and pituitary are aldosterone selective in vivo. Ameri- can J ournal of Physiology, 253: E467E471.

12. Funder J W (1995). Apparent mineralocorticoid excess. Endocrinology and M etabolism Clinics of North America, 24: 613621.

13. Ishimori K, Takeda A, Okumora S, Murai T, Inouye H \& Yasuda K (1994). Increased insulin sensitivity in patients with aldosterone producing-adenoma. Clinical Endocrinology, 41: 433-438.

14. Shimamoto $K$, Shiiki M, Ise $T$, Miyazaki $Y$, Higashiura K, Fukuoka $M$, Hirata $A$, Masuda $A$, Nakagawa $M \&$ limura $O$ (1994). Does insulin resistance participate in an impaired glucose tolerance in primary aldosteronism? J oumal of Human Hypertension, 8: 755-759.

15. Torpy DJ, Bornstein SR, Taylor W, Tauchnitz R \& Gordon RD (1999). Leptin levels are suppressed in primary aldosteronism. Hormone and M etabolic Research, 31: 533-536.

16. Conn JW (1965). Hypertension, the potassium ion and impaired glucose tolerance. New England J ournal of Medicine, 273: 1135-1143.

17. Campbell SE, Diaz-Arias AA \& Weber KT (1992). Fibrosis of the human heart and systemic organs in adrenal adenoma. Blood Pressure, 1: 149-156.

18. Sun Y, Ramires FJ \& Weber KT (1997). Fibrosis of atria and great vessels in response to angiotensin II or aldosterone infusion. Cardiovascular Research, 35: 138-147.

19. Robert V, Heymes C, Silvestre J S, Sabri A, Swynghedauw B \& Delcayre C (1999). Angiotensin AT1 receptor subtype as a cardiac target of aldosterone: role in aldosterone-salt-induced fibrosis. Hypertension, 33: 981-986.

20. Conn J W (1955). Primary aldosteronism, a new clinical syndrome. J ournal of Laboratory and Clinical Medicine, 45: 3-17.

21. Conn J W, Knopf RF \& Nesbit RM (1964). Clinical characteristics of primary aldosteronism from an analysis of 145 cases. American J ournal of Surgery, 107: 159172.

22. Kaplan NM (1992). Endocrine hypertension. In: Wilson J D \& Foster DW (Editors), Williams Textbook of Endocrinology. 8th edn. WB Saunders, Philadelphia, 707-731.

23. Streeten DHP, Tomycz $N \&$ Anderson $\mathrm{J} r$ GH (1979). Reliability of screening methods for the diagnosis of primary aldosteronism. American J ournal of Medicine, 67: 403-413.
24. Gordon RD, Stowasser M, Tunny TJ, Klemm SA \& Rutherford J C (1994). High incidence of primary hyperaldosteronism in 199 patients referred with hypertension. Clinical and Experimental Pharmacology and Physiology, 21: 315-318.

25. Gordon RD (1995). Primary aldosteronism. J ournal of Endocrinological Investigation, 18: 495-511.

26. Weisnagel SJ , Gagner M, Breton G, Pomp A, Pharand D \& Lacroix A (1996). Laparoscopic adrenalectomy. Endocrinologist, 6: 169-178.

27. Litchfield WR \& Dluhy RG (1995). Primary aldosteronism. Endocrinology and Metabolism Clinics of North America, 24: 593-612.

28. Hiramatsu K, Yamada T, Yukimura $Y$, Komiya I, Ichikawa K, Ishihara M, Nagata H \& Izumiyama T (1981). A screening test to identify aldosterone-producing adenoma by measuring plasma renin activity. Results in hypertensive patients. Archives of Internal Medicine, 141: 15891593.

29. McKenna TJ, Sequeira SJ, Heffernan A, Chambers J \& Cunningham S (1991). Diagnosis under random conditions of all disorders of the renin-angiotensin-aldosterone axis, including primary hyperaldosteronism. J ournal of Clinical Endocrinology and Metabolism, 73: 952-957.

30. Ignatowska-Switalska H, Chodakowska J , J anuszewicz W, Feltynowski T, Adamczyk M \& Lewandowski J (1997). Evaluation of plasma aldosterone to plasma renin activity ratio in patients with primary aldosteronism. J ournal of Human Hypertension, 11: 373-378.

31. Melby JC (1991). Diagnosis of hyperaldosteronism. Endocrinology and Metabolism Clinics of North America, 20: 247255.

32. Bravo EL, Turazi RC \& Dustan HP (1983). The changing clinical spectrum of primary aldosteronism. American J ournal of Medicine, 74: 641-651.

33. Hambling C, J ung RT, Gunn A, Browning MC \& Bartlett WA (1992). Re-evaluation of the captopril test for the diagnosis of primary hyperaldosteronism. Clinical Endocrinology, 36: 499-503.

34. Iwaoka T, Umeda T, Naomi S, Inoue J, Sasaki M, Yamauchi J \& Sato T (1993). The usefulness of the captopril test as a simultaneous screening for primary aldosteronism and renovascular hypertension. American J ournal of Hypertension, 6: 899906. 
35. Young J r WF \& Klee GG (1988). Primary aldosteronism: diagnostic evaluation. Endocrinology and Metabolism Clinics of North America, 17: 367-395.

36. Ulick S, Blumenfield J D, Atlas SA, Wang J Z \& Vaughan J r ED (1993). The unique steroidogenesis of the aldosteronoma in the differential diagnosis of primary aldosteronism. J ournal of Clinical Endocrinology and Metabolism, 76: 873-878.

37. Doppman J L \& Gill J J R (1996). Hyperaldosteronism: sampling the adrenal veins. Radiology, 198: 309-312.

38. Young J r WF (1997). Primary hyperaldosteronism: update on diagnosis and treatment. Endocrinologist, 7: 213-221.

39. Biglieri EG (1997). Primary aldosteronism. Current Therapy in Endocrinology and Metabolism, 6: 170-172.

40. Beckers A, Abs R \& Willems PJ (1992). Aldosterone secreting adenoma as part of multiple endocrine neoplasia type 1 (MEN I): loss of heterozygosity for polymorphic chromosome 11 deoxyribonucleic acid markers, including the MEN I locus. J ournal of Clinical Endocrinology and Metabolism, 75: 564-570.

41. Sutherland DJ A, Ruse J C \& Laidlaw J C (1966). Hypertension increased aldosterone secretion and low plasma renin activity relieved by dexamethasone. J ournal of the Canadian Medical Association, 95: 1109-1119.

42. Pascoe L, J eunemaitre $X$, Lebrethon MC, Curnow KM, Gomez-Sanchez CE, Gasc J M, Saez J M \& Corvol P (1995). Glucocorticoid-suppressible hyperaldosteronism and adrenal tumors occurring in a single French pedigree. J ournal of Clinical Investigation, 96: 2236-2246.

43. Lifton RP, Dluhy RG, Powers M, Rich GM, Cook S, Ulick S \& Lalouel J M (1992). A chimaeric 11ß-hydroxylase/aldosterone synthase gene causes glucocorticoid-remediable aldosteronism and human hypertension. Nature, 355: 262-265.

44. Ulick S \& Chu MD (1982). Hypersecretion of a new corticosteroid, 18-hydroxycortisol in two types of adrenocortical hypertension. Clinical and Experimental Hypertension, A4: 1771-1777.

45. J amieson A, Slutsker L, Inglis GC, Fraser R, White PC \& Connell J M (1995). Glucocorticoid suppressible hyperaldosteronism: effects of cross-over site and parental origin of chimaeric gene on phenotypic expression. Clinical Science, 88: 563570.

46. White PC (1997). Abnormalities of aldosterone synthesis and action in children. Current Opinion in Pediatrics, 9: 424-430.

47. Gates LJ, MacConnachie AA, Lifton RP, Hartes NE \& Benjamin N (1996). Variation of phenotype in patients with GRA. J ournal of Medical Genetics, 33: 25-28.

48. Litchfield WR, New MI, Coolidge C, Lifton RP \& Dluhy RG (1997). Evaluation of the dexamethasone suppression test for the diagnosis of glucocorticoid-remediable aldosteronism. J ournal of Clinical Endocrinology and Metabolism, 82: 3570-3573.

49. Litchfield WR, Anderson BF, Weiss RJ, Lifton RP \& Dluhy RG (1998). Intracranial aneurysm and hemorrhagic stroke in glucocorticoid-remediable aldosteronism. Hypertension, 31: 445-450.
50. Torpy DJ , Gordon RD, Lin J -P, Huggard PR, Taymans SE, Stowasser M, Chrousos GP \& Stratakis CA (1998). Familial hyperaldosteronism type-II: description of a large kindred and exclusion of the aldosterone synthase (CYP11B2) gene. J ournal of Clinical Endocrinology and Metabolism, 83: 3214-3218.

51. London N, Swales J, Hollinrake K, Bell P $\&$ Heagerty A (1992). Familial Conn's syndrome. Postgraduate Medical J ournal, 68: 976-977.

52. Greco RG, Carroll J E, Morris DJ , Grekin RJ \& Melby JC (1982). Familial hyperaldosteronism, not suppressed by dexamethasone. J ournal of Clinical Endocrinology and Metabolism, 55: 1013-1016.

53. Torpy DJ, Gordon RD \& Stratakis CA (1998). Linkage analysis of familial hyperaldosteronism type II - absence of linkage to the gene encoding the angiotensin II receptor type 1. J ournal of Clinical Endocrinology and Metabolism, 83: 1046.

54. J onsson J R, Klemm SA, Tunny TJ , Stowasser M \& Gordon RD (1995). A new genetic test for familial hyperaldosteronism type 1 aids in the detection of curable hypertension. Biochemical and Biophysical Research Communications, 207: 565-571.

55. Katoh $\mathrm{T}$, Ise $\mathrm{T}$, Takakuwa $\mathrm{H}$, Yokoyama $\mathrm{H}$ \& Kobayashi A (1997). A new variant of familial hyperaldosteronism. Proceedings of the 14th International Congress of Nephrology, Washington, DC (Abstract P1813). 